\title{
Determining the optimal magnetic resonance imaging sequences for the efficient diagnosis of temporomandibular joint disorders
}

\author{
Minjun Dong ${ }^{1 \#}$, Qi Sun ${ }^{1 \#}$, Qiang Yu ${ }^{1}$, Xiaofeng Tao ${ }^{1}$, Chi Yang ${ }^{2}$, Weiliu Qiu ${ }^{2}$ \\ ${ }^{1}$ Department of Radiology, Shanghai Ninth People's Hospital, Shanghai Jiao Tong University School of Medicine, Shanghai, China; ${ }^{2}$ Department of \\ Oral Surgery, Ninth People's Hospital, Shanghai Key Lab of Stomatology, Shanghai, China
}

\#These authors contributed equally to this work.

Correspondence to: Weiliu Qiu; Chi Yang, MD. Department of Oral Surgery, Ninth People's Hospital, Shanghai Key Lab of Stomatology, No. 639 Zhizaoju Road, Shanghai 200011, China. Email: qiuwl@cae.cn; yangchi63@hotmail.com.

Background: To compare and analyze nine MRI sequences of the TMJ and determine the optimum sequence for the rapid diagnosis of TMDs so as to develop new clinical guidelines.

Methods: Twenty young volunteers (a total of 40 joints) aged 22-26 years were recruited. Three basic sequences, $\mathrm{T}_{1}$-weighted imaging $\left(\mathrm{T}_{1} \mathrm{WI}\right), \mathrm{T}_{2}$-weighted imaging $\left(\mathrm{T}_{2} \mathrm{WI}\right)$, and proton density-weighted imaging (PDWI), together with three positions, oblique sagittal (OSag) with closed mouth, oblique coronal (OCor) with closed mouth, and OSag with opened mouth, were selected in combination for testing. In the OCor position, four regions of interest (ROIs), the condyle (C), the disc (D), the disc outside (DO), and fat (F), were analyzed. For the OSag with closed mouth position and the OSag with opened mouth position sequences, the four ROIs were the condyle (C), the disc (D), the disc ahead (DA), and the disc rear (DR). The signal-to-noise ratio (SNR), contrast-to-noise ratio (CNR), and signal intensity ratio (SIR) were calculated and analyzed using independent sample $t$-tests and one-way analysis of variance. Two senior radiologists scored the images of the nine MRI sequences subjectively and selected three optimal sequences. Using the three selected sequences, 1479 patients with anterior disc displacement with reduction (ADDwR) or anterior disk displacement without reduction (ADDwoR) were evaluated by comparing the preoperative TMJ MRI with the outcomes of the maxillofacial arthroscopy or open surgery.

Results: The $T_{1} W I$ sequence showed the highest SNR while the $T_{2} W I$ group had the lowest SNR. The ROIs of the $\mathrm{T}_{2} \mathrm{WI}$ group had the highest CNR and SIR values in the OCor and OSag sequences. In the OCor sequence, the value for the SIR F/DO group was higher than the SIR C/D and SIR C/DO values. Using subjective analysis to evaluate the quality of the scans, the highest total scores were obtained for the OSag $\mathrm{T}_{2}$ WI with opened mouth and OSag PDWI with closed mouth sequences. From the objective and subjective analysis, the three optimal sequences selected were OSag PDWI, OCor $\mathrm{T}_{2}$ WI with closed mouth, and OSag $\mathrm{T}_{2} \mathrm{WI}$ with opened mouth. In patients with anterior disc displacement, the comparisons of the surgery and the selected MRI sequences indicated that the total diagnostic accuracy of the MRI was $96.3 \%$ (1,425/1,479 cases). For patients with ADDwoR, the diagnostic accuracy was $98.5 \%(1,372 / 1,393$ cases), and for those with ADDwR it was 61.6\% (53/86 cases). There were significant differences between the ADDwoR and ADDwR groups $\left(\chi^{2}=312.92, \mathrm{P}<0.01\right)$.

Conclusions: The three optimal MRI sequences for the rapid and efficient diagnosis of TMD were determined to be OSag PDWI, OCor $\mathrm{T}_{2}$ WI with closed mouth, and OSag $\mathrm{T}_{2}$ WI with opened mouth.

Keywords: Magnetic resonance imaging (MRI); sequence; temporomandibular joint; temporomandibular joint disorders (TMDs)

Submitted Jan 09, 2020. Accepted for publication Oct 28, 2020.

doi: $10.21037 /$ qims-20-67

View this article at: http://dx.doi.org/10.21037/qims-20-67 


\section{Introduction}

Temporomandibular joint disorders (TMDs) are defined as an assorted set of clinical conditions characterized by pain and dysfunction of the masticatory muscles, the temporomandibular joint (TMJ), and the associated hard and soft tissues (1-3). Reports have shown that about $5-14 \%$ of the population experience clinical symptoms of TMD (2-4). In addition, radiographic evidence has shown that approximately $45-70 \%$ of people over the age of 65 have some degree of TMJ degeneration (5). Precise TMD diagnosis and treatment requires the assessment of structural characteristics by imaging. With the rapid development of modern medical imaging techniques, magnetic resonance imaging (MRI) has become the gold standard for the noninvasive evaluation of the TMJ region due to several factors. Firstly, there is no $\mathrm{X}$-ray radiation with MRIs and secondly, MRIs provide clear visualization of the articular disc, the retrodiscal tissue, soft-tissue structures, the cartilage, the masticatory muscles, and the synovial fluid. This is particularly important in cases where comprehensive evaluation of patients with TMD is required (6-8). However, few studies have performed systematic and detailed analysis and comparisons of the MRI sequence techniques for imaging the TMJ. To date, there is no uniform standard for the conventional sequence and no clear guidelines for the evaluation of the related TMJ anatomical structures using different sequences. Moreover, different sequences are used in different hospitals (9-19) (Table 1), and the scanning time can range from 20 to 40 minutes, requiring the patient's cooperation and tolerance for a significant period of time. Our study compared and analyzed three basic MRI sequences (including two combinations of scanning directions and mouth positions), and determined the optimum sequences for the rapid diagnosis of TMD within 10 minutes.

\section{Methods}

\section{Volunteers and patients}

A total of 20 young volunteers, including 14 females and 6 males, aged between 22 and 26 years (mean age $23.61 \pm 2.23$ years) were included in this study. From these volunteers, a total of 40 asymptomatic joints were examined. Three basic sequences, $T_{1}$-weighted imaging $\left(T_{1} W I\right), T_{2^{-}}$ weighted imaging $\left(\mathrm{T}_{2} \mathrm{WI}\right)$, and proton density-weighted imaging (PDWI), were examined (9-19). These sequences were combined with the three positions: oblique sagittal (OSag) with closed mouth, oblique coronal (OCor) with closed mouth, and OSag with opened mouth (Table 2). All volunteers underwent a simultaneous bilateral scan of the TMJ. A total of nine MRI sequences were used. The main scanning parameters of all the sequences are presented in Table 2.

A total of 1,479 patients with anterior disc displacement who were admitted to the Ninth People's Hospital, Shanghai Jiao Tong University, and the School of Medicine, between May 2018 and July 2019 were included in the study. There were 1053 females and 426 males aged between 22 and 60 years, with a mean age of $36.18 \pm 7.32$ years. All patients were diagnosed in the department of Oral Surgery or Orthodontics and were administered regular conservative treatments for several months prior to surgery. Arthroscopy or open surgery on the TMJ was conducted if patients were not responsive to conventional therapy.

None of the volunteers or patients had contraindications for MRI. The exclusion criteria were pregnancy and claustrophobia. No metal artifacts were encountered in the imaged areas. Volunteers who presented with severe bone changes in the condyle and abnormal signals in the regions of interest (ROIs) were excluded.

\section{Machine and position}

All participants underwent MRI scanning and images were produced using a 3.0T MR scanner (Ingenia, Philips Healthcare Systems, The Netherlands). The surface coils were adjusted to the center of the bilateral TMJ close to the surface of the joint, and the scanning range included the entire TMJ region. Based on the axial position, the condyle head could be viewed clearly. MRI images were acquired in both the closed mouth and opened mouth positions. Closed-mouth means the status that the mouth is closed and the posterior teeth are clenched. Opened-mouth means the status that the patient should open its mouth to the maximum. The location lines for the OSag with closed mouth position were perpendicular to the long axis of the condyle head, while the location lines for the OCor with closed mouth position were parallel to the long axis of the condyle head. The OSag position with opened mouth was adjusted according to the actual degree that the patient's mouth was opened. Patients were requested to bite onto a modal block with their teeth and keep it in place for about 2 minutes (the scanning time required for a sequence). 
Table 1 The sequences used in most studies

\begin{tabular}{|c|c|c|c|c|c|c|c|}
\hline Year & Coil & \multicolumn{4}{|c|}{ Position \& sequences } & Machine & System \\
\hline $\begin{array}{l}1998, \\
(9)\end{array}$ & $\begin{array}{l}\text { 7-cm diameter } \\
\text { surface-coil }\end{array}$ & $\begin{array}{l}\text { OSag PDWI } \\
\text { (closed mouth \& open mouth) }\end{array}$ & - & $\begin{array}{l}\text { OCor } \mathrm{T}_{1} \mathrm{WI} \\
\text { (closed mouth } \\
\text { \& open mouth) }\end{array}$ & FSE/SE & $\begin{array}{l}\text { General electric/ } \\
\text { siemens }\end{array}$ & $1.5 \mathrm{~T}$ \\
\hline $\begin{array}{l}2003 \\
(10)\end{array}$ & 3 inch Surface coil & $\begin{array}{l}\text { OSag } T_{1} \text { WI } \\
\text { (closed mouth \& open mouth) }\end{array}$ & - & $\begin{array}{l}\text { OSag } \mathrm{T}_{2} \mathrm{WI} \\
\text { (open mouth) }\end{array}$ & FSE/SE & Siemens & $1.0 \mathrm{~T}$ \\
\hline $\begin{array}{l}2007 \\
(11)\end{array}$ & $\begin{array}{l}\text { Circular-polarized } \\
\text { transmit-and-receive } \\
\text { TMJ coil }\end{array}$ & OSag PDWI (closed mouth) & $\begin{array}{l}\text { Cor PDWI } \\
\text { (closed mouth) }\end{array}$ & $\begin{array}{l}\text { Sag PDWI } \\
\text { (open mouth) }\end{array}$ & TSE & Siemens & $1.5 \mathrm{~T}$ \\
\hline $\begin{array}{l}2008 \\
(12)\end{array}$ & $\begin{array}{l}\text { 8-cm diameter surface } \\
\text { coil }\end{array}$ & $\begin{array}{l}\text { Sequential Gradient Echo } \mathrm{T}_{1} \mathrm{Wl} \\
\text { (closed mouth \& open mouth) }\end{array}$ & $\begin{array}{l}\text { Fast Stir } \mathrm{T}_{2} \mathrm{WI} \\
\text { (closed mouth \& } \\
\text { open mouth) }\end{array}$ & - & FSE/SE & General electric & $1.5 \mathrm{~T}$ \\
\hline $\begin{array}{l}2012, \\
(14)\end{array}$ & 3 inch Surface coil & OSag $\mathrm{T}_{2} \mathrm{WI}$ (closed mouth) & $\begin{array}{l}\text { OSag } \mathrm{T}_{2} \mathrm{WI} \text { fat sat } \\
\text { (closed mouth) }\end{array}$ & - & FSE & General electric & $1.5 \mathrm{~T}$ \\
\hline $\begin{array}{l}2014, \\
(15)\end{array}$ & Double loop array coil & $\mathrm{PDWI} / \mathrm{T}_{2} \mathrm{WI}$ (closed mouth) & - & $\begin{array}{l}\mathrm{PDWI} / \mathrm{T}_{2} \mathrm{WI} \\
\text { (open mouth) }\end{array}$ & $\begin{array}{l}\text { HASTE } \\
\text { (free)/TSE }\end{array}$ & Siemens & $1.5 \mathrm{~T}$ \\
\hline $\begin{array}{l}2015 \\
(16)\end{array}$ & 3 inch Surface coil & OCor $\mathrm{T}_{2} \mathrm{WI}$ (closed mouth) & $\begin{array}{l}\text { OSag PDWI } \\
\text { (closed mouth) }\end{array}$ & $\begin{array}{l}\text { OSag } \mathrm{T}_{2} \mathrm{WI} \\
\text { (open mouth) }\end{array}$ & - & $\begin{array}{l}\text { General electric } \\
\text { (signa, excite) }\end{array}$ & $1.5 \mathrm{~T}$ \\
\hline $\begin{array}{l}2016 \\
(17)\end{array}$ & $\begin{array}{l}\text { Dual surface coil Head } \\
\text { coil }\end{array}$ & OCor $\mathrm{T}_{1} \mathrm{WI}$ & OSag $T_{2} W l / T_{2} W I F S$ & OSag PDWI & $\begin{array}{l}3 \mathrm{D} \mathrm{T}_{1} \mathrm{WI} \\
\mathrm{FS}+\mathrm{C}\end{array}$ & Philips siemens & $1.5 \mathrm{~T} / 3 \mathrm{~T}$ \\
\hline
\end{tabular}

$\mathrm{T}_{2} \mathrm{WI}, \mathrm{T}_{2}$ weighted image; PdWI, proton density weighted image; $\mathrm{T}_{1} \mathrm{WI}, \mathrm{T}_{1}$ weighted image; OCor, Oblique coronal; OSag, oblique sagittal; Cor, coronal; Sag, sagittal; Ax, axial; FSE, fast spin echo; TSE, turbo spin echo; FS, fat sat; HASTE, half-Fourier acquisition single-shot turbo spin-echo.

Table 2 Acquisition parameters of the applied magnetic resonance sequences

\begin{tabular}{|c|c|c|c|c|c|c|c|c|c|}
\hline Sequences & Position & TR/TE (ms) & FOV $(\mathrm{mm})$ & Thickness/gap (mm) & Flip angle $\left(^{\circ}\right)$ & Slices & NSA & TA & Bandwidth $(\mathrm{kHz})$ \\
\hline PDWI & Closed (OCor) & $2,000 / 20$ & $110 \times 110$ & 1.5 & 90 & 16 & 2 & 2'05" & 234.8 \\
\hline $\mathrm{T}_{1} \mathrm{WI}$ & Closed (OCor) & $500 / 7.5$ & $110 \times 110$ & 1.5 & 90 & 16 & 2 & 2'03" & 226.9 \\
\hline $\mathrm{T}_{2} \mathrm{WI}$ & Closed (OSag) & $2,500 / 65$ & $110 \times 110$ & 2 & 90 & 16 & 2 & 2'07" & 206.5 \\
\hline $\mathrm{T}_{2} \mathrm{WI}$ & Opened (OSag) & $2,500 / 65$ & $110 \times 110$ & 2 & 90 & 16 & 2 & 2'07' & 206.5 \\
\hline PDWI & Opened (OSag) & $2,000 / 20$ & $110 \times 110$ & 2 & 90 & 16 & 2 & 2'05" & 234.8 \\
\hline $\mathrm{T}_{1} \mathrm{WI}$ & Opened (OSag) & $500 / 7.5$ & $110 \times 110$ & 2 & 90 & 16 & 2 & 2'03" & 226.9 \\
\hline
\end{tabular}

$\mathrm{T}_{2} \mathrm{Wl}, \mathrm{T}_{2}$ weighted image; PdWI, Proton density weighted image; $\mathrm{T}_{1} \mathrm{Wl}, \mathrm{T}_{1}$ weighted image; OCor, oblique coronal; OSag, oblique sagittal. 


\section{Statistical analysis}

All data were measured on the same display (24-inch widescreen LCD monitor, 1,920×1,200 pixels, $74.04 \mathrm{kHz}$, $60 \mathrm{~Hz}$; Hewlett-Packard Development Company, L.P., Palo Alto, CA, USA). Statistical analyses were performed using SPSS $^{\circledR}$ software v.17.0 (IBM Corp., New York, NY, USA; formerly SPSS Inc., Chicago, IL, USA). Two senior radiologists analyzed the images using a doubleblind method. To determine the inter-reader agreement on the qualitative MRI analyses, kappa statistics were used. Kappa values of $0.41-0.60$ were considered moderate agreement, values of $0.61-0.80$ were considered substantial agreement, values of $0.81-0.99$ were considered almost perfect agreement, and a value of 1.00 was considered perfect agreement. In the OCor position, four ROIs (ROI1ROI4), condyle (C), disc (D), disc outside (DO), and fat (F) were selected. For the OSag with closed mouth position and the OSag with opened mouth position sequences, four ROIs, condyle (C), disc (D), disc ahead (DA), and disc rear (DR), were selected (Figures 1,2). In addition, the background noise of each image was measured. The mean signal intensity (SI) values of each group were calculated on a workstation (Ingenia Extended WorkStation; Philips Healthcare Systems). The signal-to-noise ratio (SNR), contrast-to-noise ratio (CNR), and SI ratio (SIR) values were calculated and analyzed using an independent sample $t$-test and one-way analysis of variance for the volunteers. The calculation formulae for SNR $(20-24)$, CNR $(25,26)$, and $\operatorname{SIR}(27,28)$ were as follows:

$$
\begin{aligned}
& S N R=\bar{S}_{n} / \sigma \\
& C N R=\left|\bar{S}_{n}-\bar{S}_{N}\right| / \bar{S}_{n} \\
& \operatorname{SIR}=\bar{S}_{n} / \bar{S}_{N}
\end{aligned}
$$

$\sigma$ : standard deviation of the background noise

$\bar{S}_{n}, \bar{S}_{N}$ : mean SI value of different ROIs

Values of $\mathrm{P}<0.05$ were considered statistically significant.

Two senior radiologists reached marking consensus and scored the images of the nine sequences subjectively. The scoring criteria were as follows: 3 points for clear contrast and definite recognition of contours without artifact; 2 points for moderate contrast and probable recognition of the contour with or without a little artifact; and 1 point for low contrast and unclear contours with or without artifact. The mean value and standard deviation were measured for all nine combinations. Based on the subjective and objective evaluation methods, three optimized sequences were selected for rapid TMD MRI diagnosis. For the patient group, MRI diagnosis and operation results were compared and analyzed according to the three optimized sequences. The statistical analyses were performed using the Chisquare test. $\mathrm{P}$ values $<0.05$ were considered statistically significant.

\section{Results}

Nine imaging sequences, consisting of three OCor sequences with closed mouth and six OSag sequences (three closed mouth and three opened mouth positions), were successfully obtained from all the volunteers. All imaging sequences were feasible (Figure 1). For the repeated ROI measurements, there was good or excellent agreement [intraclass correlation (ICC), 0.78-0.99] for all ROIs in the volunteer group. For the coronal position, the four ROIs were the condyle (C), the disc (D), the outside of the disc (DO), and fat (F). For the sagittal position, the four ROIs were the condyle (C), the disc (D), the DA, and the DR.

The SNR was measured by comparing the signal of the MRI image to the background noise of the image $(29,30)$. The SNR values of the nine sequences were measured according to the imaging characteristics (Figure 2, Table 3). Among all nine combinations, the ROIs in the $\mathrm{T}_{1} \mathrm{WI}$ sequences had the highest SNR (Table 3). The disc of the $\mathrm{T}_{2} \mathrm{WI}$ sequence (in both the OCor and the OSag positions) had the lowest SNR value (Table 3).

The CNR values were obtained by calculating the deltas between the SNR values of the two tissue types. The CNR values measured for the three coronal sequences were (C-D)/C and (F-DO)/F, while the SIR values assessed for the coronal sequences were $\mathrm{C} / \mathrm{D}, \mathrm{C} / \mathrm{DO}$, and F/DO (Table 4). In the SIR comparison, it was found that the value of F/DO was higher than that of C/D and C/DO (Table 4). There were significant differences among the three OCor sequences (multiple comparison) for all five CNR and SIR comparisons $(\mathrm{P}<0.05$ for all comparisons, Table 4). In addition, the CNR and SIR values were the highest in the $\mathrm{T}_{2} \mathrm{WI}$ sequences (Table 4).

For the six sagittal sequences (three with closed mouth, C-OSag, and three with opened mouth, O-OSag), the CNR values measured were $(\mathrm{C}-\mathrm{D}) / \mathrm{C},(\mathrm{C}-\mathrm{DA}) / \mathrm{C}$, and (C-DR)/C, while the SIR values measured were C/D, C/DA, C/DR, DA/D, and DR/D (Table 5). The highest $\mathrm{CNR}$ and SIR values of the eight measured items were observed in the $\mathrm{T}_{2} \mathrm{WI}$ sequences for both closed- and opened-mouth OSag. There were significant differences 

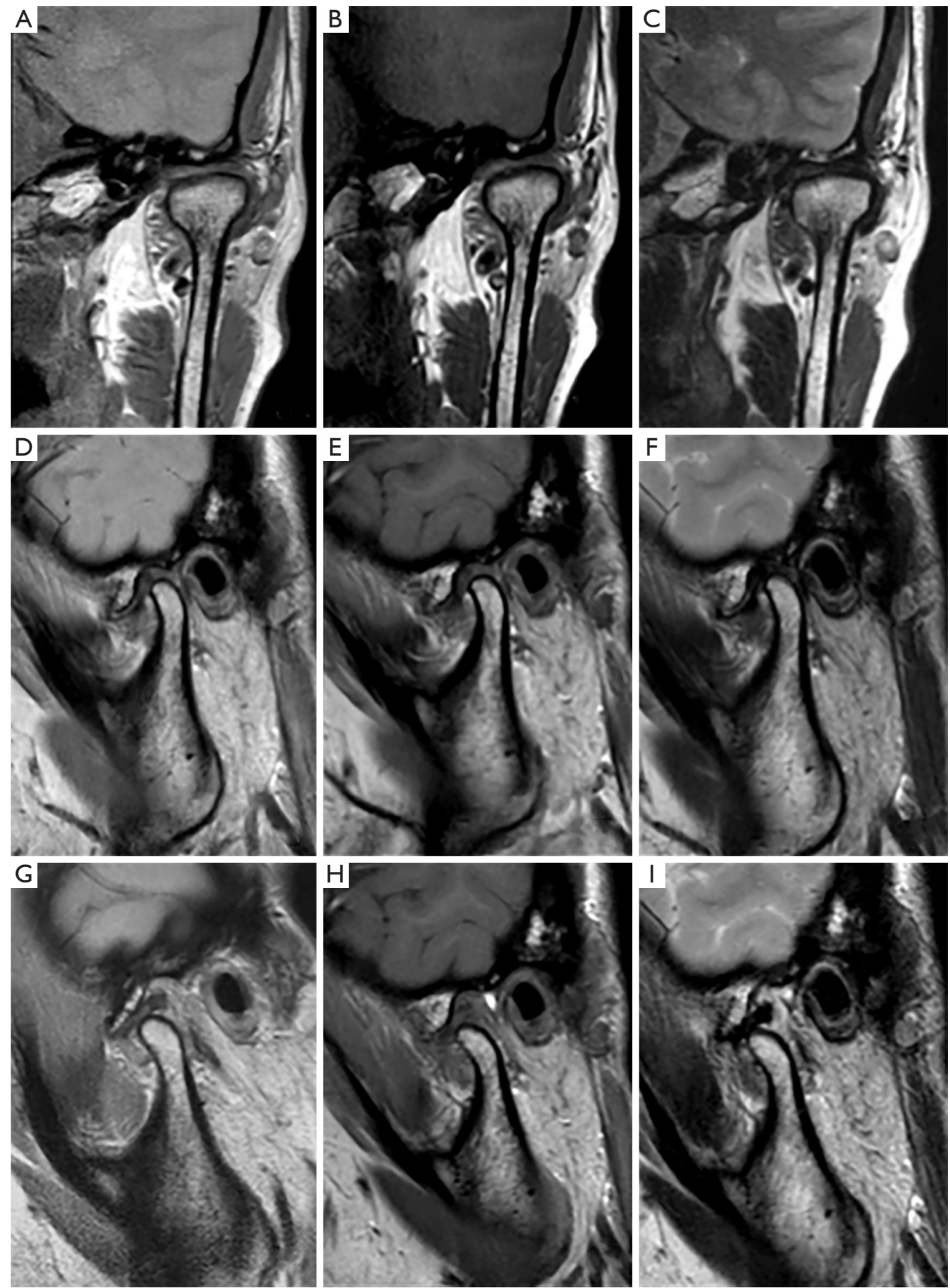

Figure 1 The nine MRI sequences (from a combination of three basic sequences, two plane positions, and two mouth postures). (A) OCor $\mathrm{T}_{1}$ WI (closed mouth); (B) OCor PDWI (closed mouth); (C) OCor $\mathrm{T}_{2} \mathrm{WI}$ (closed mouth); (D) OSag T $\mathrm{T}$ WI (closed mouth); (E) OSag PDWI (closed mouth); (F) OSag T $\mathrm{T}_{2} \mathrm{WI}$ (closed mouth); (G) OSag T $\mathrm{T}_{1} \mathrm{WI}$ (opened mouth); (H) OSag PDWI (opened mouth); (I) OSag $\mathrm{T}_{2} \mathrm{WI}$ (opened mouth). (A,B,C) show the OCor position with closed mouth; (D,E,F) show the OSag position with closed mouth; (G,H,I) show the OSag position with opened mouth. 

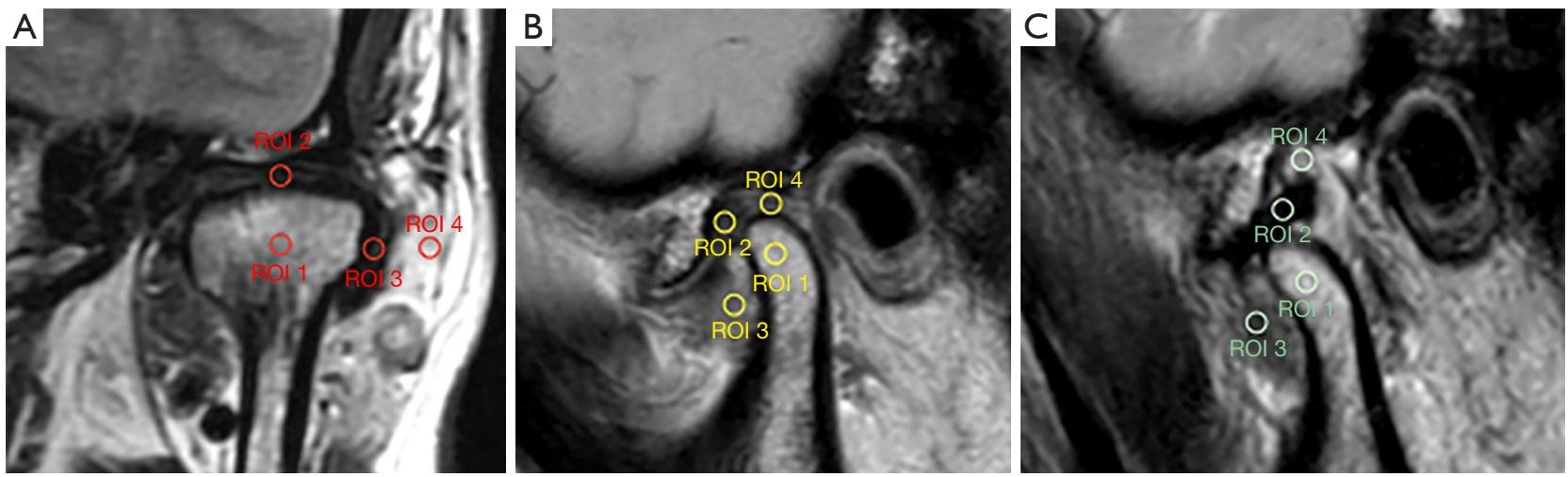

Figure 2 Regions of interest. The four regions of interest (ROIs) in the OCor position were $\mathrm{ROI}_{1}$ condyle, $\mathrm{ROI}_{2}$ disc, $\mathrm{ROI}_{3}$ disc outside, and $\mathrm{ROI}_{4}$ fat (shown with red circles). The same four ROIs examined in the OSag with closed mouth position (yellow circles) and the OSag with opened mouth position (green circles) were $\mathrm{ROI}_{1}$ condyle, $\mathrm{ROI}_{2}$ disc, $\mathrm{ROI}_{3}$ disc ahead, and $\mathrm{ROI}_{4}$ disc rear.

Table 3 The signal-to-noise values for each group

\begin{tabular}{|c|c|c|c|c|c|c|c|c|}
\hline Sequence & Condyle (ROI 1) & Disc (ROI 2) & $\begin{array}{c}\text { Disc (ROI 3) } \\
\text { (outside) }\end{array}$ & Fat (ROI 4) & Condyle (ROI 1) & Disc (ROI 2) & $\begin{array}{c}\text { Disc (ROI 3) } \\
\text { (ahead) }\end{array}$ & $\begin{array}{c}\text { Disc }(\mathrm{ROI} 4) \\
\text { (rear) }\end{array}$ \\
\hline C-OCor $\mathrm{T}_{1} \mathrm{WI}$ & $38.86 \pm 1.30$ & $15.56 \pm 1.06$ & $14.07 \pm 1.32$ & $45.87 \pm 0.80$ & - & - & - & - \\
\hline C-OCor $\mathrm{T}_{2} \mathrm{WI}$ & $30.33 \pm 1.02$ & $3.95 \pm 0.33$ & $3.67 \pm 0.25$ & $41.63 \pm 2.61$ & - & - & - & - \\
\hline C-OSag $T_{1} W I$ & - & - & - & - & $41.05 \pm 1.27$ & $18.07 \pm 0.63$ & $27.10 \pm 1.66$ & $19.76 \pm 0.53$ \\
\hline $\mathrm{C}-\mathrm{OSag} \mathrm{T}_{2} \mathrm{WI}$ & - & - & - & - & $23.71 \pm 0.61$ & $2.02 \pm 0.55$ & $9.67 \pm 0.38$ & $4.58 \pm 0.19$ \\
\hline O-OSag $\mathrm{T}_{1} \mathrm{WI}$ & - & - & - & - & $32.89 \pm 0.62$ & $12.69 \pm 0.50$ & $18.31 \pm 0.44$ & $15.79 \pm 0.40$ \\
\hline O-OSag PDWI & - & - & - & - & $22.99 \pm 0.74$ & $9.02 \pm 0.33$ & $15.98 \pm 0.17$ & $14.94 \pm 0.16$ \\
\hline
\end{tabular}

C-OCor, C-stands for closed mouth; O-OSag, O-stands for opened mouth; $\mathrm{T}_{2} \mathrm{WI}, \mathrm{T}_{2}$ weighted image; PdWI, Proton density weighted image; $T_{1}$ WI, $T_{1}$ weighted image; OCor, oblique coronal; OSag, oblique sagittal.

Table 4 The comparison results of anatomical structures in coronal sequences

\begin{tabular}{lccccc}
\hline Sequence & CNR (C-D)/C & CNR (F-DO)/F & SIR C/D & SIR C/DO & SIR F/DO \\
\hline C-OCor T 1 WI & $1.51 \pm 0.17$ & $0.69 \pm 0.28$ & $2.51 \pm 0.17$ & $2.78 \pm 0.26$ & $2.29 \pm 0.31$ \\
C-OCor PDWI & $0.96 \pm 0.08$ & $0.61 \pm 0.02$ & $1.96 \pm 0.08$ & $2.18 \pm 0.11$ & $8.3 \pm 0.71$ \\
C-OCor $\mathrm{T}_{2}$ WI & $6.76 \pm 0.88$ & $0.91 \pm 0.01$ & $7.76 \pm 0.87$ & $<0.13$ \\
P value & $<0.05$ & $<0.05$ & $<0.05$ & $<5 \pm 0.35$ \\
\hline
\end{tabular}

SIR C/D, CNR (C-D)/C: C stands for Condyle; D stands for Disc. CNR (F-DO)/F, SIR C/DO, and SIR F/DO: C stands for Condyle; F stands for Fat; DO stands for outside of Disc. $\mathrm{T}_{2} \mathrm{WI}, \mathrm{T}_{2}$ weighted image; PdWI, proton density weighted image; $\mathrm{T}_{1} \mathrm{WI}, \mathrm{T}_{1}$ weighted image; OCor, oblique coronal; OSag, oblique sagittal; c-, closed. 
Table 5 The results of contrast-to-noise and signal intensity ratio related to the anatomical structures in sagittal sequences

\begin{tabular}{lcccccccc}
\hline Sequence & CNR (C-D)/C & CNR (C-DA)/C & CNR (C-DR)/C & SIR C/D & SIR C/DA & SIR C/DR & SIR DA/D & SIR DR/D \\
\hline C-OSag T 1 WI & $1.27 \pm 0.09$ & $0.34 \pm 0.04$ & $0.52 \pm 0.01$ & $2.27 \pm 0.09$ & $1.52 \pm 0.08$ & $2.08 \pm 0.05$ & $1.50 \pm 0.09$ & $1.09 \pm 0.03$ \\
C-OSag PDWI & $2.03 \pm 0.11$ & $0.41 \pm 0.02$ & $0.54 \pm 0.01$ & $3.03 \pm 0.11$ & $1.70 \pm 0.05$ & $2.18 \pm 0.06$ & $1.78 \pm 0.05$ & $1.39 \pm 0.04$ \\
C-OSag T $_{2} W I$ & $10.74 \pm 0.36$ & $0.60 \pm 0.02$ & $0.81 \pm 0.01$ & $11.74 \pm 0.36$ & $2.45 \pm 0.11$ & $5.19 \pm 0.25$ & $4.79 \pm 0.20$ & $2.27 \pm 0.10$ \\
O-OSag T $_{1} W I$ & $1.61 \pm 0.78$ & $0.44 \pm 0.01$ & $0.52 \pm 0.01$ & $2.61 \pm 0.08$ & $1.8 \pm 0.02$ & $2.09 \pm 0.03$ & $1.45 \pm 0.05$ & $1.25 \pm 0.03$ \\
O-OSag PDWI & $1.55 \pm 0.85$ & $0.30 \pm 0.03$ & $0.35 \pm 0.26$ & $2.55 \pm 0.08$ & $1.44 \pm 0.06$ & $1.54 \pm 0.06$ & $1.77 \pm 0.02$ & $1.66 \pm 0.02$ \\
O-OSag T $\mathrm{T}_{2} \mathrm{WI}$ & $6.02 \pm 0.88$ & $0.53 \pm 0.15$ & $0.55 \pm 0.14$ & $7.02 \pm 0.09$ & $2.11 \pm 0.07$ & $2.23 \pm 0.07$ & $3.33 \pm 0.11$ & $3.15 \pm 0.09$ \\
P value & $<0.05$ & $<0.05$ & $<0.05$ & $<0.05$ & $<0.05$ & $<0.05$ & $<0.05$ & $<0.05$ \\
\hline
\end{tabular}

C-Sag, O-Sag: C-stands for closed mouth; O-stands for opened mouth. CNR (C-D)/C, CNR (C-DA)/C and CNR (C-DR)/C: C stands for Condyle; D stands for Disc; DA stands for Disc ahead; DR stands for Disc rear. SIR C/D, SIR C/DR, SIR DA/D and SIR DR/D: C stands for Condyle; D stands for Disc; DA stands for Disc ahead; DR stands for Disc rear. $\mathrm{T}_{2} \mathrm{WI}$ : T2 weighted image; PdWI: Proton density weighted image; $T_{1} W l: T_{1}$ weighted image; OCor: oblique coronal; OSag: oblique sagittal.

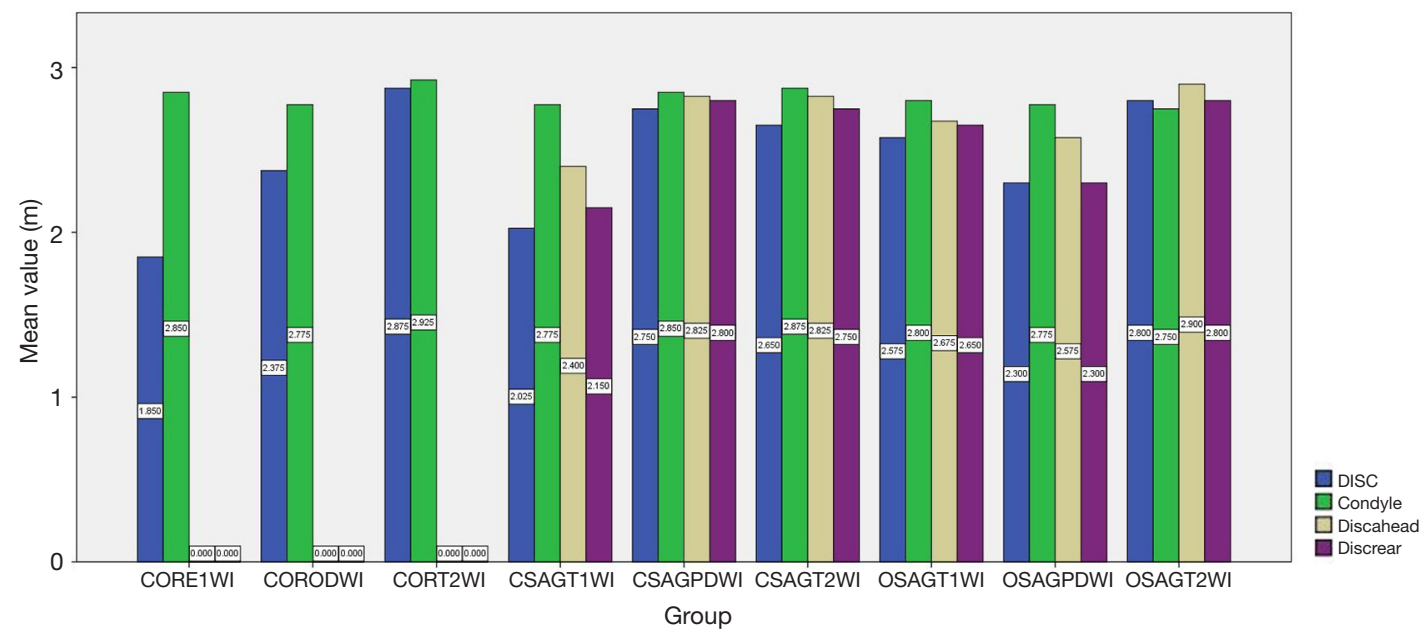

Figure 3 The histogram of the mean values for nine sequences. A histogram of the mean values was generated for nine sequences. Fourcolor histograms stand for disc (blue), condyle (green), disc ahead (light brown) and disc rear (purple) regions, respectively. The scores of T2WI with closed mouth were the highest (2.875 and 2.925 points). The C-OSag PDWI (2.75, 2.825, and 2.8 points) and O-OSag T2WI $(2.8,2.9$, and 2.8 points) sequences obtained the highest scores for articular disc, disc ahead, and disc rear, respectively; the CSag T2WI (2.875 points) and CSag PDWI (2.85 points) sequences obtained the highest scores for the condyle. C, closed; O, opened.

between the $\mathrm{T}_{2}$ WI sequence and both the $\mathrm{T}_{1} \mathrm{WI}$ and PDWI sequences, for both the closed and opened mouth positions $(\mathrm{P}<0.05$ for all comparisons, Table 5). In the closed mouth position, the CNR and SIR values of the PDWI sequence were higher than those of the $\mathrm{T}_{1} \mathrm{WI}$ sequence. In the opened mouth position, the CNR comparison indicated that the values of the $T_{1}$ WI sequence were higher than those of the PDWI sequence. Furthermore, in the SIR comparisons, with the exception of SIR DA/D and SIR DR/D, the values of the $\mathrm{T}_{1} \mathrm{WI}$ group were significantly higher than those of the PDWI group $(\mathrm{P}<0.05$, Table 5).

The quality of the nine sequences was assessed and scored subjectively. A histogram of the mean values was generated for the three OCor and six OSag sequences (Figure 3). The disc and condyle of three OCor sequences were scored. The scores of $\mathrm{T}_{2} \mathrm{WI}$ with closed mouth were the highest $(2.875$ and 2.925 points). The C-Sag PDWI and O-Sag $\mathrm{T}_{2} \mathrm{WI}$ sequences obtained the highest scores for the articular disc, DA, and DR $(2.75,2.825$, and 2.8 points, respectively, for C-Sag PDW; and 2.8, 2.9, and 2.8 points, respectively, for 
Table 6 The results of the comparison of surgery and the magnetic resonance imaging diagnosis

\begin{tabular}{|c|c|c|c|c|}
\hline Category & \multicolumn{2}{|c|}{ MR diagnosis } & Total & $P$ value \\
\hline \multicolumn{5}{|c|}{ The surgical result } \\
\hline ADDwR & 33 & 53 & 86 & $\chi^{2}=312.92 ; P<0.01$ \\
\hline Percentage & $38.4 \%$ & $61.6 \%$ & & \\
\hline Percentage & $1.5 \%$ & $98.5 \%$ & & \\
\hline Total & 54 & 1,425 & 1,479 & \\
\hline
\end{tabular}

ADDwR, anterior disc displacement with reduction; ADDwoR, anterior disk displacement without reduction.

O-Sag). The C-Sag T $\mathrm{T}_{2} \mathrm{WI}$ and C-Sag PDWI sequences obtained the highest scores for the condyle (2.875 and 2.85 points, respectively). The combinations with the highest total score of the four items were OSag $\mathrm{T}_{2} \mathrm{WI}$ in the opened mouth position (11.25 points) and OSag PDWI in the closed mouth position (11.225 points). Through the subjective and objective evaluation of data, senior oral radiologists selected three optimal sequences, OSag PDWI with closed mouth, OCor $\mathrm{T}_{2} \mathrm{WI}$ with closed mouth, and OSag $\mathrm{T}_{2} \mathrm{WI}$ with opened mouth.

The three selected optimal sequences were then used for the MR imaging of 1,479 patients with anterior disc displacement with or without reduction (ADDwR and ADDwoR, respectively). MRI scanning of the TMJ was conducted prior to maxillofacial arthroscopy or open surgery. The results of the comparison of surgery and MRI diagnosis are shown in Table 6. The total diagnostic accuracy of MRI was $96.3 \%(1,425 / 1,479$ cases $)$. The accuracy for ADDwoR was $98.5 \%$ (1,372/1,393 cases), and the accuracy for ADDwR was $61.6 \%$ (53/86 cases). There were significant differences between the ADDwoR and ADDwR groups $\left(x^{2}=312.92, \mathrm{P}<0.01\right)$.

\section{Discussion}

\section{Selection of optimized sequences}

In MRI, the SNR is conceptualized by comparing the signal of the MRI image to the background noise of the image $(29,30)$. The increase in the associated SNR could be successfully transitioned into imaging at a higher spatial resolution, allowing for the improved assessment of anatomical and pathological structures $(31,32)$. In the same sequence, CNR values can be obtained by calculating the deltas between the SNR values of two tissue types. The CNR is not directly affected by most acquisition parameters; rather, it is major influenced by SNR when the other parameters are the same, and thus CNR has become a core quality parameter for the MRI diagnosis of diseases $(31,33)$. It has been reported that quantitative alterations in the SIR could reflect the clinical features and can therefore be used as a complementary method to evaluate the correlation between the tissue signal changes and clinical outcome (27). Consequently, we selected the above three quantitative indices as objective evaluation measures to determine the optimal rapid scanning sequences in the MRI of the TMJ.

In this study, we found that objective evaluation was insufficient and that subjective analysis was preferable in real practice. Although the T1WI sequence had the highest SNR value among the three groups, it was not selected as one of the optimal TMJ imaging sequences. Two positions (OCor in the closed mouth and OSag in the opened mouth positions) of the $T_{2} W I$ sequence were selected, although the SNR values for these positions were the lowest. The $T_{2} W I$ sequence in both the coronal and sagittal positions had the highest CNR and SIR values, particularly for CNR (C-D)/ C. Therefore, the SNR value did not strongly correlate with the sequence selection, but the quantitative alterations in the CNR and SIR obtained from the calculation of the SNR correlation were of great significance in TMD diagnosis. In comparing the SIR values in the coronal position, the SIR F/DO values were higher than those of the other two groups, which indicated that the tissue signal was clearer in the lateral area of the disc-condyle complex and was thus advantageous in the diagnosis of lateral displacement of the articular disc. Similarly, in OSag imaging, the SIR 
$\mathrm{C} / \mathrm{D}$ value was the highest, particularly in the $\mathrm{T}_{2} \mathrm{WI}$ sequence, which indicated that the OSag $\mathrm{T}_{2} \mathrm{WI}$ sequence was optimal for visualization of the disc-condyle complex.

Senior radiologists subjectively evaluated all images and awarded scores of 1 to 3 for each image. The scores of the OCor $\mathrm{T}_{2} \mathrm{WI}$ with closed mouth and OSag $\mathrm{T}_{2} \mathrm{WI}$ with opened mouth sequences were the highest, which agreed with the findings of the objective evaluation. However, the scores were the highest in the OSag PDWI sequence with closed mouth in the same group, with the exception of the condyle in subjective evaluation. On this point, there are differences between subjective evaluation and objective evaluation. Therefore, after comprehensive subjective and objective evaluation, the three optimized sequences were chosen as the preferred sequences for the rapid diagnosis of TMD.

\section{Study limitations}

The present study had some limitations. First, in the experiment, we could only select patients who had surgical results and MRI scans for statistical analyses. Hence, for patients who did not undergo surgery, the accuracy of image diagnosis by using these three optimized sequences could not be determined. To further develop clinically optimized sequence versions and measurement protocols that best match different clinical situations, investigations with an increased sample size must be conducted. Second, in addition to the basic sequences investigated in this study, there are other sequences with unique advantages that were not included in this study, such as dynamic imaging, ultrashort echo time imaging, and 3D imaging. The sequence combinations chosen in this study are mainly suitable for TMD, TMJ trauma, joint ankylosis, and some tumor or tumor-like lesions such as synovial chondromatosis. For TMJ traumas, the main diagnosis from imaging is to determine whether traumatic disc displacement has occurred and whether the ligament or bilaminar region has been injured. For joint ankylosis, imaging is used to determine whether the joint disc is present or not. For synovial chondromatosis, a large amount of effusion and free bodies can be observed on $\mathrm{T}_{2} \mathrm{WI}$ with opened mouth imaging, which is the typical radiological manifestation of synovial chondromatosis. Of course, these three sequences are not sufficient for all tumor diseases in the TMJ region, and other sequences and positions need to be considered. Identifying the optimal set of sequences will be helpful for the comprehensive evaluation of TMJ diseases. Third, not all parameters and factors were considered in this study. Aside from the selection of optimized sequences, the adjustment and application of parameters are especially important. For example, Montesinos and colleagues (34) reported that the use of enhancement filters could be beneficial in the MRI imaging and diagnosis of TMJ diseases. Fourth, whether this sequence combination is significant for different clinical stages of TMJ disease requires further investigation. Moreover, whether this sequence combination could be used to guide clinical operations is yet to be determined. Lastly, due to the extended interval time between the preoperative MRI examination and the actual operation, the surgical results and MRI results differed. In the clinical group, 1,479 patients with ADDwR or ADDwoR underwent maxillofacial arthroscopy or open surgery. The accuracy of image diagnosis of $\mathrm{ADDwR}$ was $61.6 \%$, and that of $\mathrm{ADDwoR}$ was $98.5 \%$. The main reason for the inconsistency between the operation results and the MRI, and the reason ADDwoR was misdiagnosed as ADDwR, was the extended interval time between the preoperative MRI examination and the actual operation (5-8 months on average). It is possible that ADDwR was misdiagnosed as ADDwoR due to the patient's mouth not being opened to the maximum position in the opened mouth scan because of pain or an incorrectly sized mouth opening device being selected. Indeed, the time required for articular disc reduction in some patients with ADDwR was at the end of the period of mouth opening. We suggest that the interval time between TMJ MRI examination and surgery should not exceed over 1 month.

In summary, the three optimal MRI sequences selected for the rapid and efficient diagnosis of TMD were OSag PDWI, OCor $\mathrm{T}_{2} \mathrm{WI}$ with closed mouth, and OSag $\mathrm{T}_{2} \mathrm{WI}$ with opened mouth.

\section{Acknowledgments}

Funding: This study was supported by grants from the Science and Technology Commission of Shanghai Municipality, China (No. 18DZ2291100).

\section{Footnote}

Conflicts of Interest: All authors have completed the ICMJE uniform disclosure form (available at http://dx.doi. org/10.21037/qims-20-67). The authors have no conflicts of interest to declare.

Ethical Statement: This study was approved by the ethics 
board of the Shanghai Ninth People's Hospital (No. S9HIE 2017-348-T257*), and informed consent was obtained from all the patients.

Open Access Statement: This is an Open Access article distributed in accordance with the Creative Commons Attribution-NonCommercial-NoDerivs 4.0 International License (CC BY-NC-ND 4.0), which permits the noncommercial replication and distribution of the article with the strict proviso that no changes or edits are made and the original work is properly cited (including links to both the formal publication through the relevant DOI and the license). See: https://creativecommons.org/licenses/by-nc-nd/4.0/.

\section{References}

1. Ahmad M, Schiffman EL. Temporomandibular joint disorders and orofacial pain. Dent Clin North Am 2016;60:105-24.

2. Chantaracherd P, John MT, Hodges JS, Schiffman EL. Temporomandibular joint disorders' impact on pain, function, and disability. J Dent Res 2015;94:79S-86S.

3. Liu F, Steinkeler A. Epidemiology, diagnosis, and treatment of temporomandibular disorders. Dent Clin North Am 2013;57:465-79.

4. Hongxing L, Astrøm AN, List T, Nilsson IM, Johansson A. Prevalence of temporomandibular disorder pain in Chinese adolescents compared to an age-matched Swedish population. J Oral Rehabil 2016;43:241-8.

5. Sumit Yadav, Yun Yang, Eliane H. Dutra, Jennifer L. Robinson, Sunil Wadh. Temporomandibular Joint Disorders in the Elderly and Aging Population. J Am Geriatr Soc 2018;66:1213-7.

6. Sun Q, Dong MJ, Tao XF, Yu Q, Li KC, Yang C. Dynamic MR imaging of temporomandibular joint: an initial assessment with fast imaging employing steadystate acquisition sequence. Magn Reson Imaging 2015;33:270-5.

7. Eberhard L, Giannakopoulos NN, Rohde S, Schmitter M. Temporomandibular joint (TMJ) disc position in patients with TMJ pain assessed by coronal MRI. Dentomaxillofac Radiol 2013;42:20120199.

8. Manoliu A, Spinner G, Wyss M, Erni S, Ettlin DA, Nanz D, Ulbrich EJ, Gallo LM, Andreisek G. Quantitative and qualitative comparison of MR imaging of the temporomandibular joint at 1.5 and $3.0 \mathrm{~T}$ using an optimized high-resolution protocol. Dentomaxillofac Radiol 2016;45:20150240.
9. Foucart JM, Carpentier P, Pajoni D, Marguelles-Bonnet R, Pharaboz C. MR of 732 TMJs: anterior, rotational, partial and sideways disc displacements. Eur J Radiol 1998;28:86-94.

10. Huh JK, Kim HG, Ko JY. Magnetic resonance imaging of temporomandibular joint synovial fluid collection and disk morphology. Oral Surg Oral Med Oral Pathol Oral Radiol Endod 2003;95:665-71.

11. Emshoff R, Rudisch A. Temporomandibular joint internal derangement and osteoarthrosis: are effusion and bone marrow edema prognostic indicators for arthrocentesis and hydraulic distention? J Oral Maxillofac Surg 2007;65:66-73.

12. Manfredini D, Basso D, Arboretti R, Guarda-Nardini L. Association between magnetic resonance signs of temporomandibular joint effusion and disk displacement. Oral Surg Oral Med Oral Pathol Oral Radiol Endod 2009;107:266-71.

13. Bertram S, Moriggl A, Rudisch A, Emshoff R. Structural characteristics of bilateral temporomandibular joint disc displacement without reduction and osteoarthrosis are important determinants of horizontal mandibular and vertical ramus deficiency: a magnetic resonance imaging study. J Oral Maxillofac Surg 2011;69:1898-904.

14. Barchetti F, Stagnitti A, Glorioso M, Al Ansari N, Barchetti G, Pranno N, Montechiarello S, Pasqualitto E, Sartori A, Marini A, Gigli S. Static and dynamic MR imaging in the evaluation of temporomandibular disorders. Eur Rev Med Pharmacol Sci 2014;18:2983-7.

15. Shen P, Sun Q, Xu W, Zhen J, Zhang S, Yang C. The fate of autogenous free fat grafts in the human temporomandibular joint using magnetic resonance imaging. J Craniomaxillofac Surg 2015;43:1804-8.

16. Krohn S, Gersdorff N, Wassmann T, Merboldt KD, Joseph AA, Buergers R. Real-time MRI of the temporomandibular joint at 15 frames per second-A feasibility study. Eur J Radiol 2016;85:2225-30.

17. Inarejos Clemente EJ, Tolend $M$, Junhasavasdikul $T$, Stimec J, Tzaribachev N, Koos B, Spiegel L, Moineddin $\mathrm{R}$, Doria AS. Qualitative and semi-quantitative assessment of temporomandibular joint MRI protocols for juvenile idiopathic arthritis at 1.5 and 3.0 T. Eur J Radiol 2018;98:90.

18. Takahara N, Nakagawa S, Sumikura K, Kabasawa Y, Sakamoto I, Harada H. Association of temporomandibular joint pain according to magnetic resonance imaging findings in temporomandibular disorder patients. J Oral Maxillofac Surg 2017;75:1848-55. 
19. Angenete OW, Augdal TA, Jellestad S, Rygg M, Rosendahl K. Normal magnetic resonance appearances of the temporomandibular joints in children and young adults aged 2-18 years. Pediatr Radiol 2018;48:341-9.

20. Sun Q, Dong MJ, Tao XF, Jiang MD, Yang C. Selection and application of coils in temporomandibular joint MRI. Dentomaxillofac Radiol 2020;49:20190002.

21. Komlosi P, Altes TA, Qing K, Mooney KE, Miller GW, Mata JF, de Lange EE, Tobias WA, Cates GD Jr, Mugler III JP. Signal-to-noise ratio, T2, and T2* for hyperpolarized helium-3 MRI of the human lung at three magnetic field strengths. Magn Reson Med 2017;78:1458-63.

22. Riederer SJ, Borisch EA, Froemming AT, Grimm RC, Kawashima A, Mynderse LA, Trzasko JD. Improved performance of prostate DCE-MRI using a 32 coil vs. 12-coil receiver array. Magn Reson Imaging 2017;39:15-23.

23. Chen Q, Quijano CV, Mai VM, Krishnamoorthy SK, Li W, Storey P, Edelman RR. On improving temporal and spatial resolution of 3D contrast-enhanced body MR angiography with parallel imaging. Radiology 2004;231:893-9.

24. Jutras JD, Wachowicz K, Gilbert G, De Zanche N. SNR efficiency of combined bipolar gradient echoes: comparison of three-dimensional FLASH, MPRAGE, and multiparameter mapping with VFA-FLASH and MP2RAGE. Magn Reson Med 2017;77:2186-202.

25. Nordmeyer-Massner JA, Wyss M, Andreisek G, Pruessmann KP, Hodler J. In vitro and in vivo comparison of wrist MR imaging at 3.0 and 7.0 tesla using a gradient echo sequence and identical eight-channel coil array designs. J Magn Reson Imaging 2011;33:661-7.

26. Wintersperger BJ, Runge VM, Biswas J, Reiser MF, Schönberg SO. Brain tumor enhancement in MR imaging at 3 Tesla: comparison of SNR and CNR gain using TSE and GRE techniques. Invest Radiol 2007;42:558-63.

Cite this article as: Dong M, Sun Q, Yu Q, Tao X, Yang C, Qiu W. Determining the optimal magnetic resonance imaging sequences for the efficient diagnosis of temporomandibular joint disorders. Quant Imaging Med Surg 2021;11(4):13431353. doi: 10.21037/qims-20-67
27. Kim TH, Ha Y, Shin JJ, Cho YE, Lee JH, Cho WH. Signal intensity ratio on magnetic resonance imaging as a prognostic factor in patients with cervical compressive myelopathy. Medicine (Baltimore) 2016;95:e4649.

28. Yamoto M, Iwazaki T, Takeuchi K, Sano K, Fukumoto K, Takahashi T, Nomura A, Ooyama K, Sekioka A, Yamada Y, Urushihara N. The fetal lung-to-liver signal intensity ratio on magnetic resonance imaging as a predictor of outcomes from isolated congenital diaphragmatic hernia. Pediatr Surg Int 2018;34:161-8.

29. Marijke Welvaert, Yves Rosseel. On the definition of signal-to-noise ratio and contrast-to-noise ratio for fMRI data. PLoS One 2013;8:e77089.

30. Parrish TB, Gitelman DR, LaBar KS, Mesulam MM. Impact of signal-to-noise on functional MRI. Magn Reson Med 2000;44:925-32.

31. Kuhn FP, Spinner G, Del Grande F, Wyss M, Piccirelli M, Erni S, Pfister P, Ho M, Sah BR, Filli L, Ettlin DA. MR imaging of the temporomandibular joint: comparison between acquisitions at 7.0 $\mathrm{T}$ using dielectric pads and 3.0 T. Dentomaxillofac Radiol 2017;46:20160280.

32. Laader A, Beiderwellen K, Kraff O, Maderwald S, Wrede K, Ladd ME, Lauenstein TC, Forsting M, Quick HH, Nassenstein K, Umutlu L. 1.5 versus 3 versus 7 Tesla in abdominal MRI: a comparative study. PLoS One 2017;12:e0187528.

33. Honda E, Sasaki T, Simm FC, Maruyama K. An optimized fast protocol for magnetic resonance imaging of the temporomandibular joint. Dentomaxillofac Radiol 2001;30:126-30.

34. Montesinos GA, de Castro Lopes SLP, Trivino T, Sánchez JA, Maeda FA, de Freitas CF, Costa ALF. Subjective analysis of the application of enhancement filters on magnetic resonance imaging of the temporomandibular joint. Oral Surg Oral Med Oral Pathol Oral Radiol 2019;127:552-9. 